\title{
Abdominal Signs and Symptoms of COVID-19 in Children
}

\author{
Rana Saleh ${ }^{1}$, Hamid Rahimi ${ }^{1}$, Niloofar Javadi ${ }^{2}$, Sheida Amini ${ }^{1}$, Shima Saeidi ${ }^{1}$ and Zahra \\ Pourmoghaddas, ${ }^{1 *}$ \\ ${ }^{1}$ Pediatric Infectious Disease Department, Isfahan University of Medical Sciences, Isfahan, Iran \\ ${ }^{2}$ Student Research Committee, School of Medicine, Isfahan University of Medical Sciences, Isfahan, Iran \\ "Corresponding author: Pediatric Infectious Disease Department, Isfahan University of Medical Sciences, Isfahan, Iran. Email: za.pourmoghaddas@gmail.com
}

Received 2020 July 07; Revised 2020 August 26; Accepted 2020 September 03.

\begin{abstract}
COVID-19 is an ongoing pandemic that is not known well. According to our current knowledge, although respiratory manifestations are the most common symptoms of COVID-19, but non-pulmonary manifestations are also commonly reported, such as rashes, appendicitis, volvulus, diverticulitis, and mesenteric lymphadenitis. In this case series, we introduced four children with COVID-19 who presented with gastrointestinal symptoms.
\end{abstract}

Keywords: COVID-19, SARS-CoV-2, Gastrointestinal Symptoms, Pediatric, Acute Abdomen

\section{Introduction}

In December 2019, a disease presented mostly by respiratory symptoms begun to spread around the world from Wuhan, Hubei Province, China. The World Health Organization announced "COVID-19" as the name of this new disease (COVID-19). It causes by severe acute respiratory syndrome coronavirus-2 (SARS-CoV-2) (1).

According to case series on pediatrics infected with SARS-CoV-2, common symptoms of COVID-19 in children are fever, dry cough, and dyspnea (2). Meanwhile, some epidemiological studies have also mentioned to gastrointestinal symptoms, including diarrhea, vomiting, gastrointestinal bleeding, and abdominal pain (3). According to the best knowledge of the authors, this is the first case report in children with acute abdomen signs.

In this article, we reported four children infected with COVID-19 who, during their hospital stay, developed acute abdominal pain, which led to the diagnosis of acute abdomen.

\section{Case Presentation}

To observe ethical principles, written informed consent was obtained from parents of children who participated in the current study. Also, the privacy of the participants was respected.

\subsection{Case 1}

A four-month-old boy with a history of hospitalization due to severe pneumonia who was using bi-level positive airway pressure (BiPAP) machine developed fever, poor feeding, and dyspnea (Table 1). His arterial oxygen saturation was $75 \%$ (Table 2 ).

He was suffering from ontogenesis imperfecta and had a history of contact with a COVID-19 confirmed case while hospitalized at the pediatric intensive care unit (PICU). Physical examination revealed subcostal retraction and crackle in both lungs. Laboratory examination showed increased LDH and decreased hemoglobin concentration, while other tests were normal (Table 3). The diagnosis of COVID-19 was confirmed by detection of SAR-CoV-2 RNA and typical lesions in the chest after performing computed tomography (CT) (Figure 1). Following the Iranian pediatric protocol, he was treated with hydroxychloroquine and lopinavir/ritonavir. During the hospitalization, a new-onset abdominal tenderness and bilious vomiting appeared. The abdominal X-ray showed a coffee-bean view in the left upper quadrant (Figure 1). By considering signs and symptoms, the patient underwent laparotomy with suspicion of volvulus. Laparotomy revealed several large lymph nodes in the peritoneum (Figure 1).

\subsection{Case 2}

A10-year-old boy was admitted to the pediatric COVID19 center with loss of consciousness and its associated respiratory distress (Table 1). During physical examination, 


\begin{tabular}{|c|c|c|c|c|}
\hline Parameters & Case 1 & Case 2 & Case 3 & Case 4 \\
\hline Age & 4 months & 10 years & 5 years & 45 days \\
\hline Sex & Boy & Boy & Girl & Boy \\
\hline Fever & Yes & No & Yes & No \\
\hline $\begin{array}{l}\text { Abdominal signs and } \\
\text { symptoms }\end{array}$ & $\begin{array}{l}\text { Abdominal tenderness and } \\
\text { bilious vomiting }\end{array}$ & $\begin{array}{l}\text { Abdominal pain, tenderness } \\
\text { and guarding }\end{array}$ & $\begin{array}{l}\text { Abdominal pain, tenderness } \\
\text { and guarding and vomiting }\end{array}$ & $\begin{array}{l}\text { Oral intolerance, abdominal } \\
\text { distention and irritability }\end{array}$ \\
\hline Imaging finding & Coffee-bean view in X-ray & $\begin{array}{l}\text { Appendicitis in } \\
\text { ultrasonography }\end{array}$ & $\begin{array}{l}\text { Appendicitis in } \\
\text { ultrasonography }\end{array}$ & Coffee-bean view in X-ray \\
\hline
\end{tabular}

\begin{tabular}{|c|c|c|c|c|}
\hline Parameters & Case 1 & Case 2 & Case 3 & Case 4 \\
\hline $\mathrm{PR}, / \mathrm{min}$ & 123 & 120 & 110 & 162 \\
\hline $\mathbf{R R}, / \mathrm{min}$ & 38 & 30 & 28 & 45 \\
\hline $\mathrm{O}_{2}$ sat with BIPAP, $\%$ & 92 & 96 & 96 & 98 \\
\hline Axillary temperature, ${ }^{\circ}$ Celsius & 37.5 & 36.8 & 37.7 & 37 \\
\hline CRT, $s$ & $<2$ & $<2$ & $<2$ & $<2$ \\
\hline AVPU & A & $\mathrm{U}$ & A & A \\
\hline Ability to drink & Not good & Not good & Good & Not good \\
\hline
\end{tabular}

Abbreviations: AVPU, a scale to record level of consciousness including alert (A), verbal (V), pain (P), unresponsive (U); CRT, capillary refill time; $\mathrm{O}_{2}$ sat, oxygen saturation; $\mathrm{PR}$, pulse rate; $\mathrm{RR}$, respiratory rate.

Table 3. Laboratory Data in 4 Cases

\begin{tabular}{|c|c|c|c|c|}
\hline Markers & Case 1 & Case 2 & Case 3 & Case 4 \\
\hline WBC count, cells $/ \mu \mathbf{L}$ & 5500 & 11400 & 6100 & 14000 \\
\hline Lymphocyte percent, \% & 58.9 & 27 & 8 & 66.9 \\
\hline $\mathrm{Hb}, \mathrm{g} / \mathrm{dL}$ & 8.4 & 11.5 & 12.7 & 15 \\
\hline PLT count, cells/mL & 245000 & 252000 & 63000 & 65000 \\
\hline AST, $\mathbf{U} / \mathbf{L}$ & - & - & - & 110 \\
\hline ALT, U/L & - & - & - & 80 \\
\hline $\mathbf{A l b}, \mathbf{g} / \mathbf{d L}$ & 4.3 & - & 2.7 & - \\
\hline Qualitative CRP & ++ & + & + & + \\
\hline ESR, $\mathbf{m m} / \mathbf{h}$ & 26 & 38 & 26 & 11 \\
\hline $\mathbf{P C T}, \mu \mathbf{g} / \mathbf{L}$ & 2.5 & - & - & 0.5 \\
\hline BUN, mg/dL & 13 & 11 & - & 14 \\
\hline $\mathrm{Cr}, \mathbf{m g} / \mathbf{d L}$ & 0.4 & 0.5 & - & 0.6 \\
\hline LDH, U/L & 1080 & - & - & - \\
\hline
\end{tabular}

Abbreviations: Alb, albumin; ALT, alanine aminotransferase; AST, aspartate aminotransferase; BUN, blood urea nitrogen; Cr, creatinine; CRP, C-reactive protein; ESR, erythrocyte sedimentation rate; Hb, hemoglobin; LDH, lactic acid dehydrogenase; PCT, procalcitonin; PLT, platelet; WBC, white blood cell.

his oxygen saturation was $80 \%$, measured by a pulse oximeter, the respiratory rate was 45 per minute, and subcostal and intercostal retraction was also observed (Table 2). Laboratory exams showed leukocytosis, lymphopenia, and elevated C-reactive protein (CRP) (Table 3). Due to his respi- ratory distress, intubation was needed. The chest $\mathrm{CT}$ scan was highly suggestive for COVID-19 (Figure 2). Besides, the result of the polymerase chain reaction (PCR) for SARS-CoV2 RNA was positive. Thus, he was treated according to the Iranian pediatric protocol for treating COVID-19. 

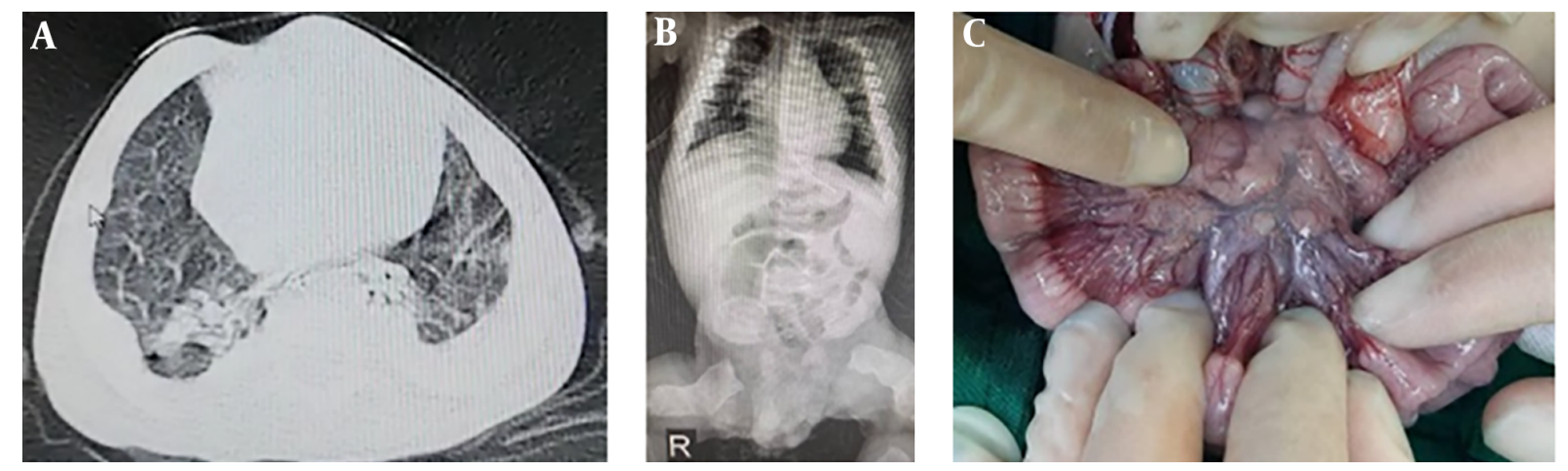

Figure 1. Images from case 1: A, Axial slice of chest CT scan at admission time showing two-sided ground-glass opacity and crazy paving pattern; B, abdominal X-ray showing a coffee-bean view in the left upper quadrant; C, several large lymph nodes in the peritoneum during laparotomy.
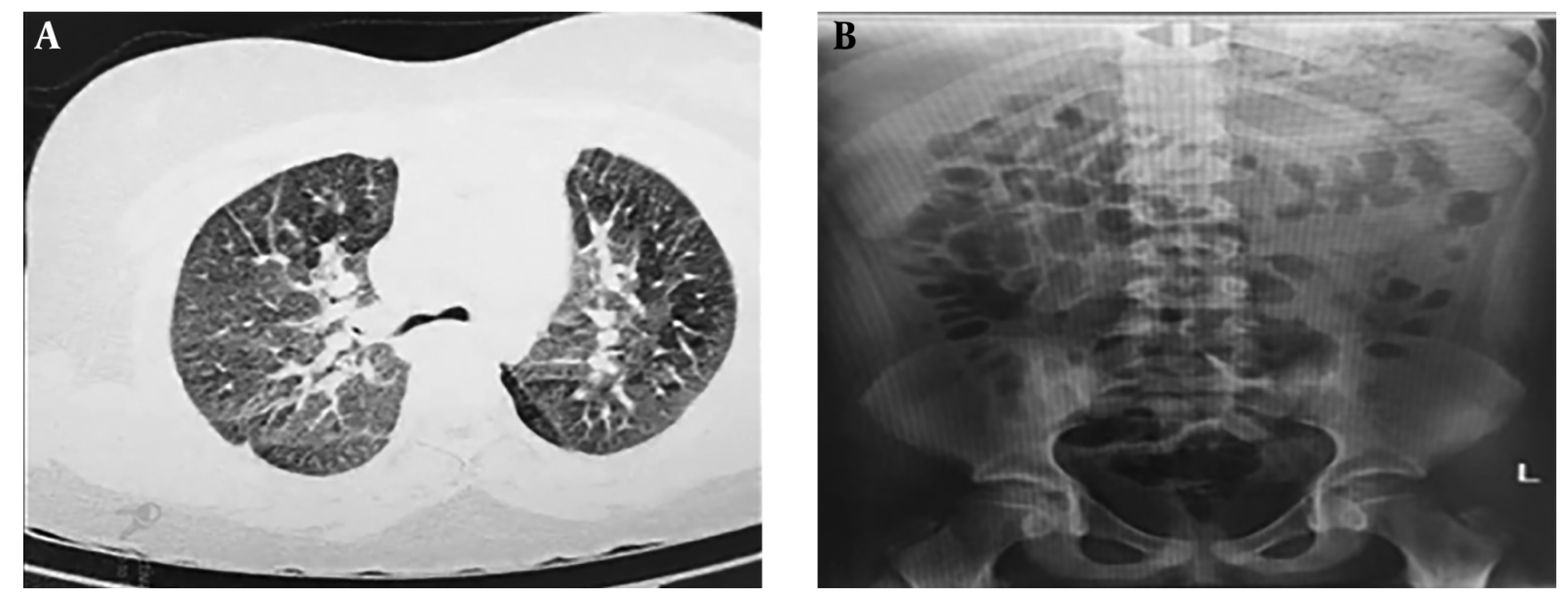

Figure 2. Two different image modalities from case 2: A, Axial slice of chest CT scan at admission time showing two-sided ground-glass opacity confirming COVID-19 pneumonia; B, no abnormal finding was observed in abdominal X-ray.

After the respiratory condition has stabilized, he was extubated and went on a Bi-PAP machine. Also, the symptoms gradually disappeared. He developed abdominal pain, guarding, and tenderness during the hospital stay. As the first step of a diagnostic approach, an abdominal X-ray was done, and no abnormal finding was observed (Figure 2 ). In the next step, the ultrasonography was performed, and the patient was diagnosed with appendicitis. Therefore, he went on an appendectomy. During the surgery, the patient's Meckel's diverticulum and peritoneal lymphadenopathy were identified and resected.

\subsection{Case3}

A five-year-old girl who suffered from fever, cough, myalgia, and abdominal pain was admitted to the pediatric COVID-19 center(Table1). Her grandmothers were sus- pected for COVID-19, and she had a history of contact with them.

In physical examination, tachypnea, 40 per minute, subcostal retraction, and abdominal tenderness were detected (Table 2). Her laboratory examination revealed thrombocytopenia, lymphopenia, decreased serum albumin, and elevated CRP (Table 3 ). The chest CT scan showed typical lesions of COVID-19 (Figure 3). According to signs, symptoms, and suggestive chest CT scan, she was a probable case of COVID-19. Therefore, her therapy begun based on the Iranian pediatric protocol. Since she had vomiting, abdominal pain, tenderness, and guarding, an ultrasound was performed, and appendicitis was established as the probable diagnosis. Serous secretion around the appendix and several large lymph nodes were observed during the appendectomy (Figure 3). 

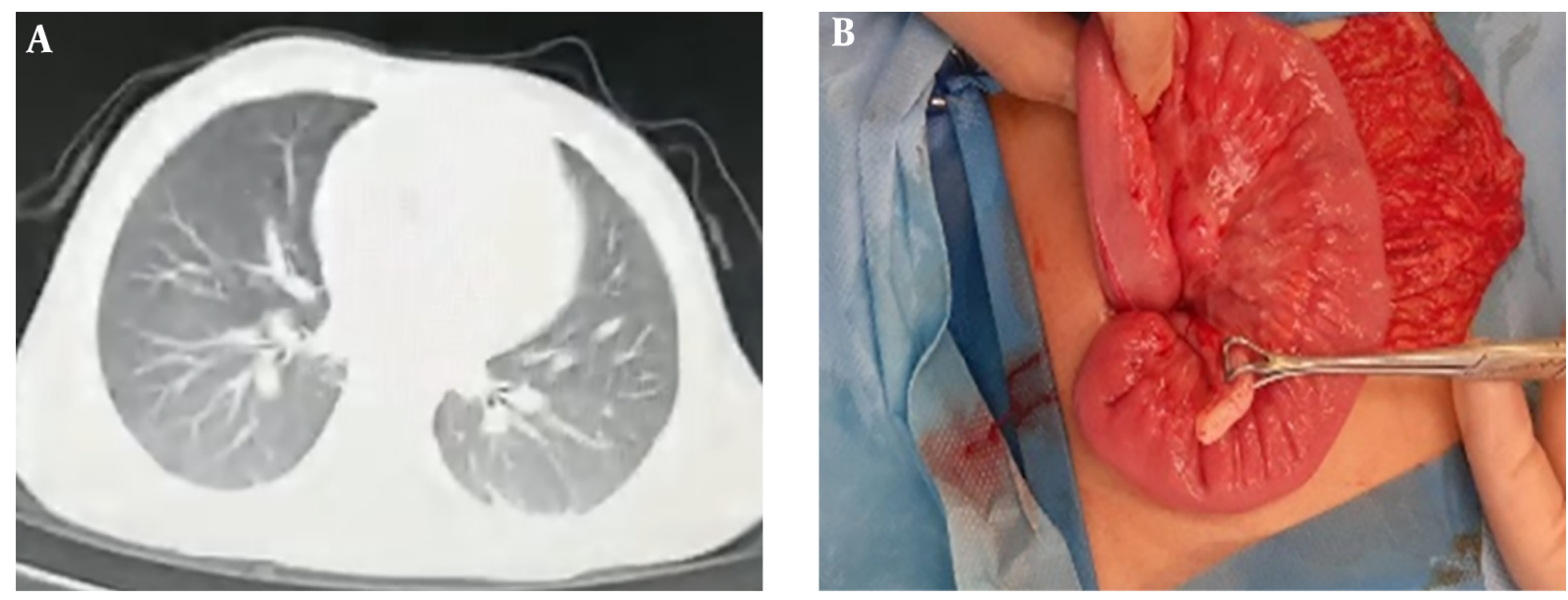

Figure 3. Two different images from case 3: A, Axial slice of chest CT scan at admission time revealing two-sided ground glass-opacity due to COVID-19 pneumonia; B, severa large lymph nodes in the peritoneum during appendectomy.

\subsection{Case4}

A 45 day-old-infant living in a nursery was admitted to the pediatric COVID-19 center with cough and respiratory distress (Table 1). He was a known case of cerebral palsy and a seizure disorder. In his physical examination, decreased arterial oxygen saturation, $75 \%$ in room air, nasal flaring, and suprasternal retraction were seen (Table 2). In laboratory study, thrombocytopenia, normal lymphocyte count, and elevated CRP was detected (Table 3). Diagnosis of COVID-19 was made after a positive result of the PCR test, and typical chest CT scan lesions (Figure 4). The patient received antiviral and antibacterial therapies, according to the Iranian pediatric protocol. On the third day of hospitalization, he developed oral intolerance, abdominal distention, and irritability. In his abdominal X-ray, a coffeebean view was seen (Figure 4). Because of the severe illness and critical condition of the patient, the medical team decided to exclude surgery from therapeutic choices; the patient was treated by medical management. Gradually with the improvement of the patient's respiratory condition, the gastrointestinal symptoms disappeared.

\section{Discussion}

When the WHO declared a global pandemic on 11 March 2020, the seriousness of the COVID-19 was revealed. Until 4 May 2020, there were 3,435,894 confirmed cases of COVID19 and 239,604 deaths $(1,4)$.

According to recent studies, most of the COVID-19 patients are adults with predominant symptoms of cough, fever, fatigue, sputum production, shortness of breath, myalgia or arthralgia, sore throat, headache, nausea, vomiting, and diarrhea $(5,6)$.

Our literature review showed that respiratory-related symptoms are the most common symptom of COVID-19 among adults (6). In addition, COVID-19 patients may manifest other presentations, such as cardiac and gastrointestinal signs and symptoms. Based on a systematic review and meta-analysis study, only $12 \%$ of COVID-19 patients develop gastrointestinal symptoms (1). For instance, acute abdomen rarely occurs in adults infected with COVID-19 and even more uncommon in children (7-9).

On 11 February 2020, the Chinese Center for Disease Control and Prevention reported 44,672 confirmed cases of COVID-19, who only 416 (0.9\%) of them were children aged 0 to 9 years and $549(1.2 \%)$ were adolescents aged 10 to 19 years (10).

According to our literature review about abdominal signs in children with COVID-19, a case series by CabreroHernandez et al. (8) reported five children infected with SARS-CoV-2 who were suspected of an acute abdomen. This article demonstrated that all patients had fever and abdominal pain, $60 \%$ presented with vomiting and diarrhea (simultaneously), and the rest were suffering only from vomiting (8). Another study conducted in May 2020 in the United Kingdom on the gastrointestinal presentations of COVID-19 has reported eight children presented at a single center in the UK with symptoms of atypical appendicitis. The most common gastrointestinal symptoms were abdominal pain, diarrhea, and vomiting (11). In the present study, out of four cases, only 2 ( 1 and 3 ) developed fever ( Table 2). Also, cases 2 and 3 complained of abdominal pain, while cases 1 and 4 , who could not complain about abdom- 


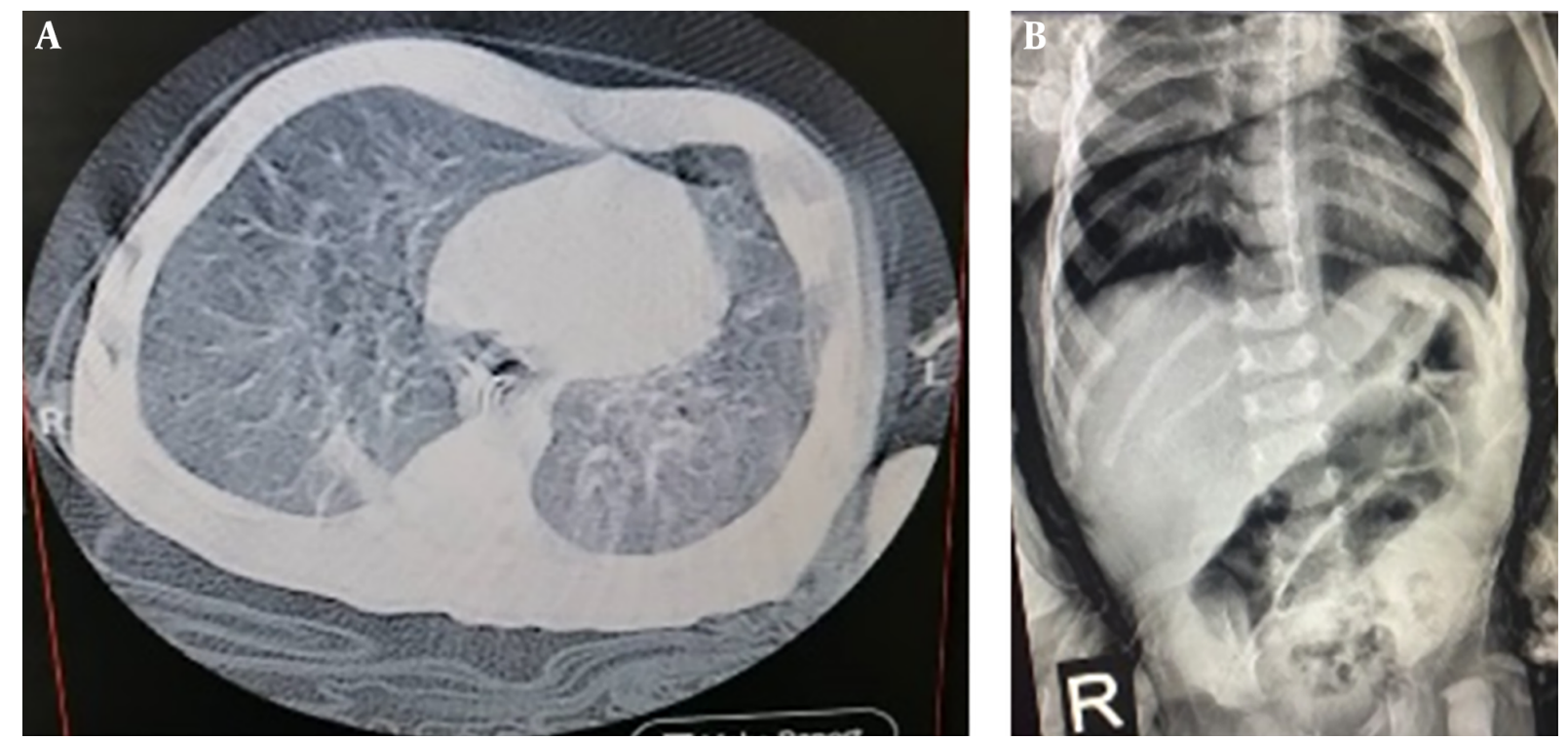

Figure 4. Different image modalities from case 4: A, Axial slice of chest CT scan at admission time revealing two-sided ground-glass opacity and crazy paving pattern; B, abdominal X-ray showing a coffee-bean view.

inal pain, showed oral intolerance, abdominal distension, and tenderness.

The next step of the diagnostic approach is abdominal imaging, i.e., ultrasonography or CT scan. Also, a study conducted in the UK recommended abdominal imaging when investigating for possible appendicitis in children with COVID-19, and all children in this study had imaging confirming terminal ileitis. Cabrero-Hernandez et al. (8) used imaging tools for participating patients, and the results showed inflammation of the intestine without surgically treatable disease. In this study, the abdominal X-ray of cases number one four revealed a coffee-bean view (Figures 1 and 4); ultrasonography was also performed for cases 2 and 3 and suggested appendicitis.

Still, there is no confirmed treatment for COVID-19 infection and its complications. In the study performed by Cabrero-Hernandez's, the results of the CT scan didn't suggest surgically treatable disease. Therefore, all cases received non-surgical treatment, including antibiotic therapy, lopinavir-ritonavir, tocilizumab, heparin, and hydroxychloroquine. All cases were discharged from PICU with a good overall health condition. Also, in the UK study, no surgical intervention was required, and all children were treated with non-surgical treatment. In our study, case 1 underwent laparotomy because of suspected volvulus. Also, appendectomy was done for cases 2 and 3 due to the results of ultrasonography. Diffuse peritoneal lymphadenopathies were detected for all three cases (Figures 1 and 3). Due to the critical condition of case 4, medical therapy was provided instead of surgery. All patients were also discharged with good condition.

\subsection{Conclusions}

According to our current knowledge, respiratory signs and symptoms are the most common presentations of the SARS-CoV-2. In this study, we reported four children presented with gastrointestinal symptoms that were later diagnosed with COVID-19. Therefore, pediatricians need to distinguish GI symptoms from COVID-19 manifestations.

\section{Footnotes}

Authors' Contribution: RS, NJ, SA, and SS contributed to the diagnoses of these cases in the hospital. ZP and HR contributed to the supervising the team in diagnosis and all authors contributed to the writing of the manuscript.

Conflict of Interests: The authors declare no conflict of interest.

Ethical Approval: This study was approved by the ethics Committee of the Isfahan University of Medical Sciences (code: IR.MUI.MED.REC.1399.024198264).

Funding/Support: This research received no specific grant from any funding agency in the public, commercial, or not-for-profit sectors. 
Informed Consent: According to ethical principles, written informed consent (ICF) was obtained from all children's parents, and the authors were committed to signing the confidentiality and privacy terms regarding the identity of the patients and their data.

\section{References}

1. Chang TH, Wu JL, Chang LY. Clinical characteristics and diagnostic challenges of pediatric COVID-19: A systematic review and meta-analysis. J Formos Med Assoc. 2020;119(5):982-9. doi: 10.1016/j.jfma.2020.04.007. [PubMed: 32307322]. [PubMed Central: PMC7161491].

2. Zimmermann $P$, Curtis N. Coronavirus Infections in Children Including COVID-19: An Overview of the Epidemiology, Clinical Features, Diagnosis, Treatment and Prevention Options in Children. Pediatr Infect Dis J. 2020;39(5):355-68. doi: 10.1097/INF.0000000000002660. [PubMed: 32310621]. [PubMed Central: PMC7158880].

3. Pan L, Mu M, Yang P, Sun Y, Wang R, Yan J, et al. Clinical Characteristics of COVID-19 Patients With Digestive Symptoms in Hubei, China: A Descriptive, Cross-Sectional, Multicenter Study. Am J Gastroenterol. 2020;115(5):766-73. doi:10.14309/ajg.0000000000000620. [PubMed: 32287140]. [PubMed Central: PMC7172492].

4. World Health Organization (WHO). Situation report - 105 coronavirus disease 2019 (COVID-19). 2020, [cited 2020 May 5]. Available from: www.who.int/emergencies/diseases/novel-coronavirus2019/situation-reports.

5. Guan WJ, Ni ZY, Hu Y, Liang WH, Ou CQ, He JX, et al. Clinical Characteristics of Coronavirus Disease 2019 in China. N Engl J
Med. 2020;382(18):1708-20. doi: 10.1056/NEJMoa2002032. [PubMed: 32109013]. [PubMed Central: PMC7092819].

6. Lovato A, de Filippis C. Clinical Presentation of COVID-19: A Systematic Review Focusing on Upper Airway Symptoms. Ear Nose Throat J. 2020:145561320920762. doi: 10.1177/0145561320920762. [PubMed: 32283980]

7. Kang Y, Chen T, Mui D, Ferrari V, Jagasia D, Scherrer-Crosbie M, et al. Cardiovascular manifestations and treatment considerations in COVID-19. Heart. 2020;106(15):1132-41. doi: 10.1136/heartjnl-2020317056. [PubMed: 32354800]. [PubMed Central: PMC7211105].

8. Cabrero-Hernandez M, Garcia-Salido A, Leoz-Gordillo I, AlonsoCadenas JA, Gochi-Valdovinos A, Gonzalez Brabin A, et al. Severe SARS-CoV-2 Infection in Children With Suspected Acute Abdomen: A Case Series From a Tertiary Hospital in Spain. Pediatr Infect Dis J. 2020;39(8):e195-8. doi: 10.1097/INF.0000000000002777. [PubMed: 32467457].

9. Saeed U, Sellevoll HB, Young VS, Sandbaek G, Glomsaker T, Mala T. Covid-19 may present with acute abdominal pain. Br J Surg. 2020;107(7):e186-7. doi: 10.1002/bjs.11674. [PubMed: 32343396]. [PubMed Central: PMC7267330].

10. Epidemiology Working Group for Ncip Epidemic Response. [The epidemiological characteristics of an outbreak of 2019 novel coronavirus diseases (COVID-19) in China]. Zhonghua Liu Xing Bing Xue Za Zhi. 2020;41(2):145-51. Chinese. doi: 10.3760/cma.j.issn.02546450.2020.02.003. [PubMed: 32064853].

11. Tullie L, Ford K, Bisharat M, Watson T, Thakkar H, Mullassery D, et al. Gastrointestinal features in children with COVID-19: an observation of varied presentation in eight children. Lancet Child Adolesc Health. 2020;4(7):e19-20. doi: 10.1016/S2352-4642(20)30165-6. [PubMed: 32442420]. [PubMed Central: PMC7237361]. 